\title{
Aegilops tauschii draft genome sequence reveals a gene repertoire for wheat adaptation
}

\begin{abstract}
Jizeng Jia ${ }^{1 *}$, Shancen Zhao ${ }^{2,3 *}$, Xiuying Kong ${ }^{1 *}$, Yingrui $\mathrm{Li}^{2 *}$, Guangyao Zhao ${ }^{1 *}$, Weiming $\mathrm{He}^{2 *}$, Rudi Appels ${ }^{4 *}$, Matthias Pfeifer ${ }^{5}$, Yong Tao ${ }^{2}$, Xueyong Zhang ${ }^{1}$, Ruilian Jing ${ }^{1}$, Chi Zhang ${ }^{2}$, Youzhi Ma ${ }^{1}$, Lifeng $\mathrm{Gao}^{1}$, Chuan $\mathrm{Gao}^{2}$, Manuel Spannagl ${ }^{5}$,

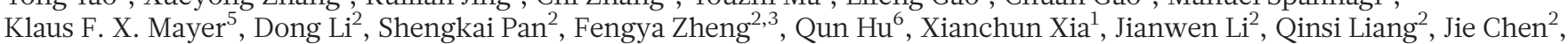
Thomas Wicker ${ }^{7}$, Caiyun Gou ${ }^{2}$, Hanhui Kuang ${ }^{6}$, Genyun $\mathrm{He}^{2}$, Yadan Luo ${ }^{2}$, Beat Keller ${ }^{7}$, Qiuju Xia ${ }^{2}$, Peng Lu², Junyi Wang ${ }^{2}$, Hongfeng Zou ${ }^{2}$, Rongzhi Zhang ${ }^{1}$, Junyang $\mathrm{Xu}^{2}$, Jinlong $\mathrm{Gao}^{2}$, Christopher Middleton ${ }^{7}$, Zhiwu Quan ${ }^{2}$, Guangming Liu ${ }^{8}$, Jian Wang ${ }^{2}$, International Wheat Genome Sequencing Consortium†े, Huanming Yang ${ }^{2}$, Xu Liu ${ }^{1}$, Zhonghu He ${ }^{1,9}$, Long Mao \& Jun Wang ${ }^{2,10,11}$
\end{abstract}

\begin{abstract}
About 8,000 years ago in the Fertile Crescent, a spontaneous hybridization of the wild diploid grass Aegilops tauschii $(2 n=14$; DD) with the cultivated tetraploid wheat Triticum turgidum $(2 n=4 x=28$; $\mathrm{AABB}$ ) resulted in hexaploid wheat ( $T$. aestivum; $2 n=6 x=42$; AABBDD $)^{1,2}$. Wheat has since become a primary staple crop worldwide as a result of its enhanced adaptability to a wide range of climates and improved grain quality for the production of baker's flour ${ }^{2}$. Here we describe sequencing the Ae. tauschii genome and obtaining a roughly 90 -fold depth of short reads from libraries with various insert sizes, to gain a better understanding of this genetically complex plant. The assembled scaffolds represented $83.4 \%$ of the genome, of which $65.9 \%$ comprised transposable elements. We generated comprehensive RNA-Seq data and used it to identify 43,150 protein-coding genes, of which 30,697 (71.1\%) were uniquely anchored to chromosomes with an integrated high-density genetic map. Whole-genome analysis revealed gene family expansion in Ae. tauschii of agronomically relevant gene families that were associated with disease resistance, abiotic stress tolerance and grain quality. This draft genome sequence provides insight into the environmental adaptation of bread wheat and can aid in defining the large and complicated genomes of wheat species.
\end{abstract}

We selected Ae.tauschii accession AL8/78 for genome sequencing because it has been extensively characterized genetically (Supplementary Information). Using a whole genome shotgun strategy, we generated $398 \mathrm{~Gb}$ of high-quality reads from 45 libraries with insert sizes ranging from $200 \mathrm{bp}$ to $20 \mathrm{~kb}$ (Supplementary Information). A hierarchical, iterative assembly of short reads employing the parallelized sequence assembler SOAPdenovo ${ }^{3}$ achieved contigs with an N50 length (minimum length of contigs representing $50 \%$ of the assembly) of $4,512 \mathrm{bp}$ (Table 1). Paired-end information combined with an additional $18.4 \mathrm{~Gb}$ of Roche/454 long-read sequences was used sequentially to generate 4.23-Gb scaffolds (83.4\% were non-gapped contiguous sequences) with an N50 length of $57.6 \mathrm{~kb}$ (Supplementary Information). The assembly represented $97 \%$ of the $4.36-\mathrm{Gb}$ genome as estimated by $K$-mer analysis (Supplementary Information). We also obtained 13,185 Ae.tauschii expressed sequence tag (EST) sequences using Sanger sequencing, of which 11,998 (91\%) could be mapped to the scaffolds with more than 90\% coverage (Supplementary Information).

To aid in gene identification, we performed RNA-Seq $(53.2 \mathrm{~Gb}$ for a $117-\mathrm{Mb}$ transcriptome assembly) on 23 libraries representing eight tissues including pistil, root, seed, spike, stamen, stem, leaf and sheath
(Supplementary Information). Using both evidence-based and de novo gene predictions, we identified 34,498 high-confidence protein-coding loci. FGENESH ${ }^{4}$ and GeneID models were supported by a $60 \%$ overlap with either our ESTs and RNA-Seq reads, or with homologous proteins. More than $76 \%$ of the gene models had a significant match (Evalue $\leq 10^{-5}$; alignment length $\geq 60 \%$ ) in the GenBank nonredundant database. An additional 8,652 loci were predicted as lowconfidence genes as a result of incomplete gene structure or limited expression data support (Supplementary Information). We also predicted a total of 2,505 transfer RNA, 358 ribosomal RNA, 35 small nuclear RNA and 78 small nucleolar RNA genes (Supplementary Information).

We found that more than $65.9 \%$ of the Ae.tauschii genome was composed of different transposable element (TE) families (Supplementary Information). About $5 \times 10^{6}$ Illumina reads of Ae.tauschii were mapped to hexaploid wheat repetitive sequences and we found that a comparable percentage of reads (more than 62.3\%) could be classified as part of a TE sequence (Supplementary Fig. 6). This estimate is similar to that derived from a previous survey of Roche $/ 454$ sequences ${ }^{5}$. There were 410 different TE families, of which the 20 most abundant contributed more than $50 \%$ of the Ae.tauschii genome (Supplementary Table 9). A single peak of increased insertion activity was estimated to occur about 3-4 Myr ago by measuring the similarity of the assembled LTR retrotransposons (Supplementary Information), suggesting that the expansion of the Ae. tauschii genome was relatively recent and coincided with the abrupt climate change during the Pliocene Epoch ${ }^{6}$.

We constructed a high-density genetic map using an $\mathrm{F}_{2}$ population of 490 individuals derived from a cross between the Ae.tauschii accessions Y2280 and AL8/78. The map, whose total length was 1059.8 centimorgans (cM), consisted of 151,083 single nucleotide polymorphism (SNP) markers developed by restriction-site-associated DNA (RAD) tag sequencing technology (Supplementary Fig. 13). Together with bin-mapped wheat ESTs ${ }^{7}$, SNPs and tags ${ }^{8}$, the genetic map was used to align 30,303 scaffolds ( $1.72 \mathrm{~Gb}$; 30,697 genes) to chromosomes (Supplementary Information). The Ae.tauschii genes and scaffolds were also anchored to barley ${ }^{9}$ and Brachypodium chromosome maps ${ }^{10}$ (Fig. 1 and Supplementary Fig. 17). Calculation of $K_{\mathrm{a}} / K_{\mathrm{s}}$ ratios (the ratio of non-synonymous substitutions to synonymous substitutions) for pairs of conserved orthologous genes showed that the average values between Ae.tauschii and barley (20,892 genes), Brachypodium (17,231 genes), rice (16,370 genes) and sorghum $(18,623$ genes) were $0.2214,0.1888,0.1736$ and 0.1726 , respectively,

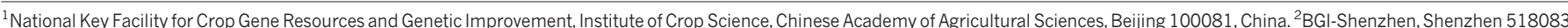

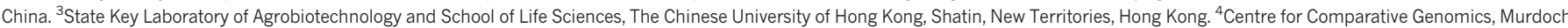

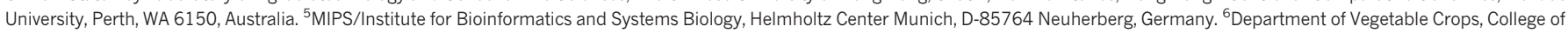

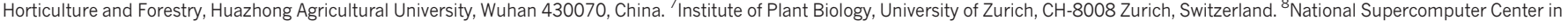

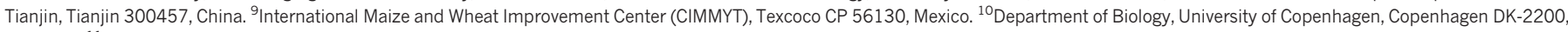
Denmark. ${ }^{11}$ King Abdulaziz University, Jeddah 21589, Saudi Arabia.

*These authors contributed equally to this work.

$\dagger$ A list of participants and their affiliations appears in the Supplementary Information. 
Table 1 | Overall statistics of sequencing and genome assembly

\begin{tabular}{|c|c|c|c|c|c|c|c|}
\hline Assembly process & Library insert size (bp) & Read length (bp) & Effective data (Gb) & N50 (bp) & N50 number & Total length $(\mathrm{Mb})$ & Gaps (Mb) \\
\hline Contig assembly & $167-764$ & $44,75,100,150$ & 270 & 4,521 & 179,145 & 3,528 & - \\
\hline Scaffolding & $2,000-20,000$ & $44,49,90$ & 128 & 58,011 & 19,405 & 4,244 & 1,122 \\
\hline Gap closure & $167-764$ & $\begin{array}{l}44,75,100,150 \\
114-263\end{array}$ & $\begin{array}{l}270 \\
65\end{array}$ & 57,585 & 19,455 & 4,229 & 701 \\
\hline
\end{tabular}

* Reads from 454 sequencing platform.

which indicated that most gene lineages evolved under purifying selection in Ae. tauschii. A total of 628 genes exhibited $K_{\mathrm{a}} / K_{\mathrm{s}}$ ratios of more than 0.8 when compared with the other four species, indicating potential positive selection (innermost circle of Fig. 1). These genes were assigned to a wide range of molecular functions by using Gene Ontology (GO) analyses (Supplementary Table 14).

Ae. tauschii proteins were clustered with those of Brachypodium, rice, sorghum and barley (full-length complementary DNAs), and formed 23,202 orthologous groups (at least two members; Supplementary Information). In total, we identified 11,289 (barley/Ae.tauschii) and 14,675 (Brachypodium/Ae.tauschii) orthologous gene pairs. We found that 8,443 gene groups contained sequences from all five grass genomes, and 234 were specific to Pooideae (Ae.tauschii, Brachypodium and barley) and 587 were specific to Triticeae (Ae.tauschii and barley) (Fig. 2a). Enrichment analyses of both Pfam domains and GO terms showed that genes encoding NBS-LRR (nucleotide-binding-site leucine-rich repeat) proteins were over-represented in Ae.tauschii relative to Brachypodium and rice ${ }^{11,12}$ (Supplementary Information). These observations are consistent with those reported in a recent study $^{13}$. A total of 1,219 Ae.tauschii genes were similar to NBS-LRR genes ( $\mathrm{R}$ gene analogues (RGAs) $)^{11,14}$ (Supplementary Information). This number is double that in rice (623) and sixfold that in maize $(216)^{12}$, indicating that the RGA family has substantially expanded in Ae. tauschii. We mapped 878 RGAs (72\%) to specific positions across wheat chromosomes by using molecular marker-genome sequence alignment, which provides a large number of potential disease resistance loci for further investigation.

We found more genes for the cytochrome P450 family in Ae.tauschii (485) than in sorghum (365), rice (333), Brachypodium (262) or maize (261). This family of genes is important for abiotic stress response, especially in biosynthetic and detoxification pathways ${ }^{15}$. Using 178 manually curated cold-acclimation-related genes such

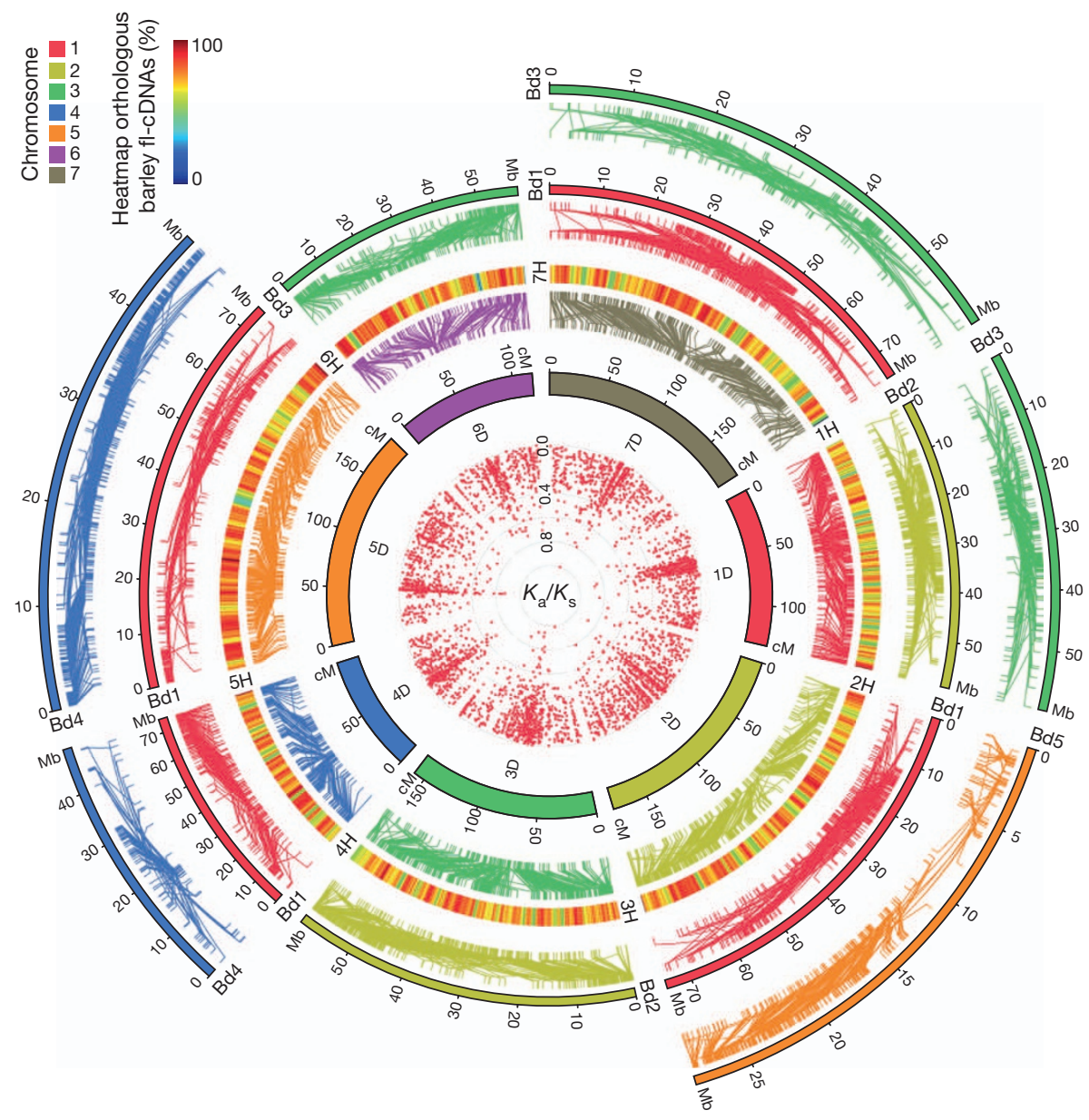

Figure 1 Comparative analysis of Ae. tauschii ordered scaffolds versus barley and Brachypodium. The inner circle represents the seven Ae.tauschii chromosomes scaled according to the genetic map incorporating genome scaffolds. Red points show the $K_{\mathrm{a}} / K_{\mathrm{s}}$ ratios between anchored Ae. tauschii genes and their putative orthologues in Brachypodium. Moving outwards, the second circle compares Ae. tauschii against the seven barley chromosomes ${ }^{9}$. The heatmaps show the density distribution of barley cDNA loci that are aligned with Ae. tauschii genes. The outer two circles illustrate Brachypodium chromosomes according to conserved synteny with Ae. tauschii. The coloured lines below each chromosome identify putative orthologous gene pairs between Ae. tauschii genes, barley genes and Brachypodium genes. 


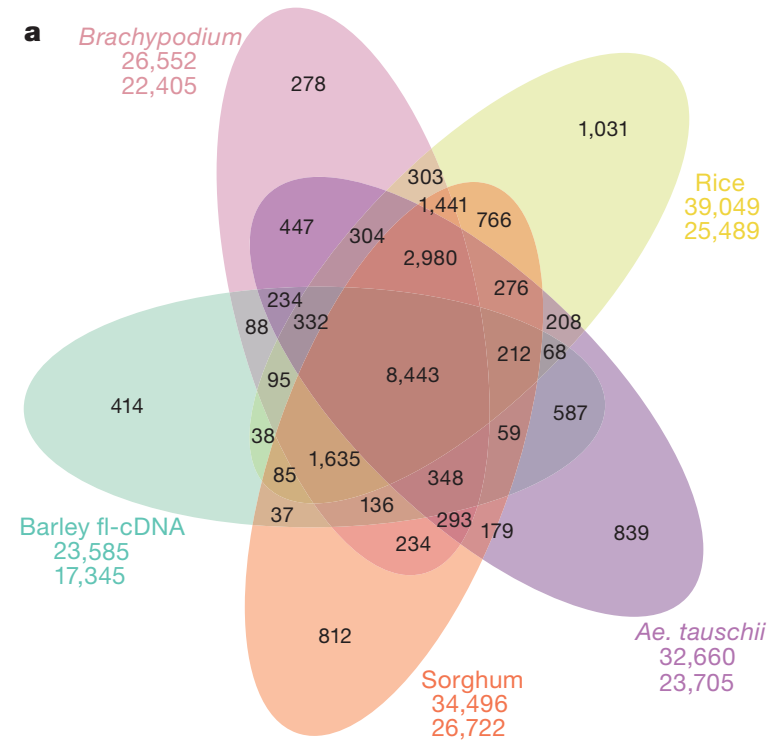

Figure $2 \mid$ Ae. tauschii gene families and transcription factors.

a, Distribution of orthologous gene families in Ae. tauschii, Brachypodium, sorghum, rice and barley. The number of gene families is represented in each intersection of the Venn diagram. The first number under the species name indicates the total number of genes annotated for a particular species, and the

as the CCAAT-binding factor $(\mathrm{CBF})$ transcription factors ${ }^{16}$, lateembryogenesis-abundant proteins (LEA) and osmoprotectant biosynthesis proteins (Supplementary Information) as queries, we identified 216 cold-related genes in the Ae.tauschii genome, in contrast to 164 genes in Brachypodium, 132 in rice, 159 in sorghum and 148 in maize. Some of these genes were specific to Ae. tauschii or to Pooideae, including those encoding ice-recrystallization inhibition protein 1 precursor, DREB2 transcription factor $\alpha$ isoform and cold-responsive LEA/RABrelated COR protein. Expression analysis of RNA-Seq data showed that most of these Ae.tauschii-specific and Pooideae-specific genes were constitutively expressed in Ae. tauschii (Supplementary Fig. 23). In addition, 1,489 transcription factors (TFs) in 56 families were identified by using Pfam DNA-binding domains (Supplementary Information). Ae.tauschii had an excess of such TFs as MYB-related genes (103, in contrast with 66 in Brachypodium and 95 in maize), and these are also thought to be involved in various stress responses ${ }^{17}$. The M-type MADS-box genes (58, in contrast with 23 in Brachypodium and 34 in maize) are involved in regulation in plant reproduction ${ }^{18}$ (Fig. $2 b$ and Supplementary Table 18). ARACNe ${ }^{19}$ co-expression analysis using RNA-Seq data predicted an expression network of 1,283 interactions (Supplementary Fig. 25), in which 13 TFs were associated with the expression of drought tolerance genes ${ }^{20}$ (Supplementary Table 20).

We predicted a total of 159 (133 families) previously undescribed microRNAs (Supplementary Information), and identified segmental and tandem duplications in 42 members of the miR2118 family that were organized into two groups on 15 scaffolds (Supplementary Fig. 26). The miR399 family, which is involved in the regulation of inorganic phosphate homeostasis in rice ${ }^{21}$, was expanded (20 members in Ae.tauschii, compared with 11 in rice and 10 in maize), and may contribute to the ability of Ae.tauschii to grow in low-nutrient soils. The expansion of the miR2275 family (eight members in Ae.tauschii, compared with two in rice and four in maize) may contribute to the enhanced disease resistance of Ae.tauschii because phased short interfering RNAs initiated by miR2275 have been implicated in these activities ${ }^{22}$.

The Ae.tauschii genome served as the source for many grain quality genes in hexaploid wheat, creating a step improvement in the formation of the elastic dough essential for bread making ${ }^{2}$. Grain quality genes include high-molecular-weight glutenin subunits (HMW-GS),

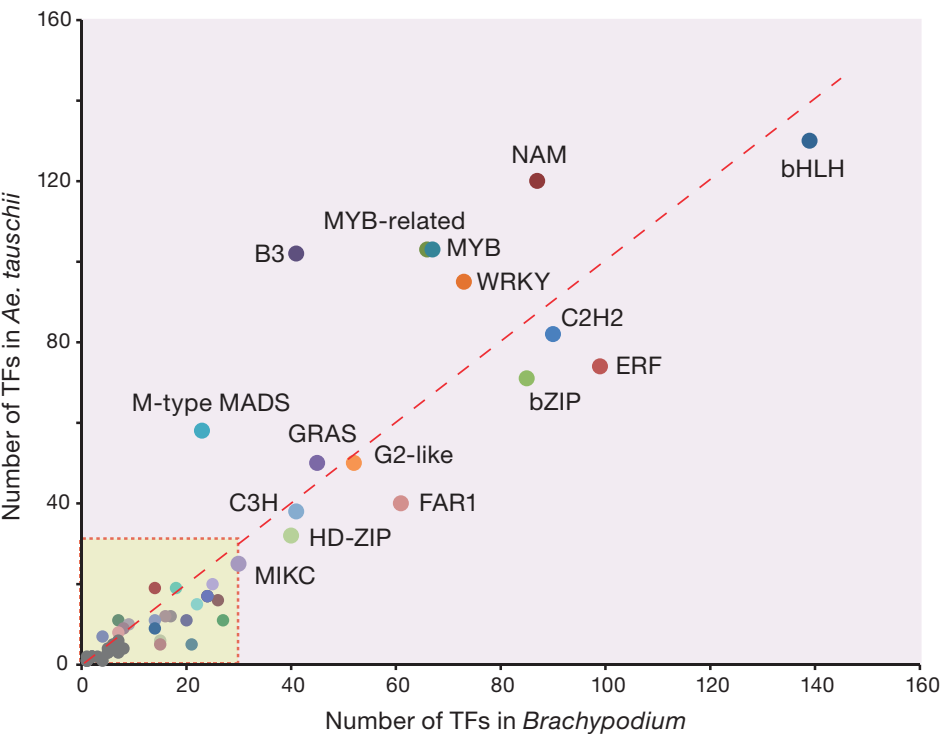

second indicates the number of genes in groups for that organism. The difference between the two accounts for singleton genes that were not present in any cluster. b, The composition of transcription factors (TFs) in Ae. tauschii and Brachypodium composed of more than 30 members.

low-molecular-weight glutenin subunits (LMW-GS) ${ }^{23}$, grain texture proteins (GSP; puroindolines) ${ }^{24}$ and storage protein activator (SPA) ${ }^{25}$. We identified two $H M W$-GS genes, five $L M W$-GS genes, one Pina gene, two Pinb genes, one GSP gene and one SPA gene in the Ae.tauschii genome sequence (Supplementary Information). As has been shown for the Hardness ( $\mathrm{Ha}$ ) locus ${ }^{24}$, the GSP, Pina and Pinb genes were also organized in a cluster. RNA-Seq analysis showed that these grain quality genes were expressed predominantly in seeds (Supplementary Fig. 29).

The anchoring of more than $40 \%$ of the scaffold sequences to four genetic maps and to syntenic regions of other sequenced grass species provided a structural framework for integrating multiple maps by using shared markers (Fig. 1 and Supplementary Information). The co-localization of genes in scaffolds and genetically mapped quantitative trait loci (QTLs) will directly support map-based gene cloning. On chromosome 2D, for example, the locations of 33 QTLs or genes were integrated with scaffold information (http://ccg.murdoch.edu.au/ cmap/ccg-live/) (Fig. 3 and Supplementary Information). Alignment of the Ae.tauschii genetic map with the wheat $2 \mathrm{D}$ consensus genetic map was unambiguous, with the exception of some single crossovers that were probably due to repetitive elements (dotted lines in Fig. 3). The genome sequence also provided the basis for the identification of more than 860,126 simple sequence repeats (SSRs), with trimers (37.7\%) and tetramers (27.5\%) as the most abundant SSR types (Supplementary Information). Together with the 711,907 SNPs identified by resequencing a roughly fivefold coverage of a second accession, Y2280 (Supplementary Information), the genomic resources reported here will promote map-based gene cloning and marker-assisted selection in wheat.

With its high base accuracy and nearly complete set of gene sequences, the Ae.tauschii draft genome sequence provides an essential reference for studying $\mathrm{D}$ genome diversity by re-sequencing additional accessions. Over the past half century, the introduction of new $\mathrm{D}$ genome diversity into synthetic wheat has been a major effort to expand bread wheat genetic diversity and to create environmentally resilient lines ${ }^{26,27}$. The Ae.tauschii genome sequence should aid in identifying new elite alleles for agriculturally important traits to alleviate the worsening plight of global climate and environment changes $^{27}$. 


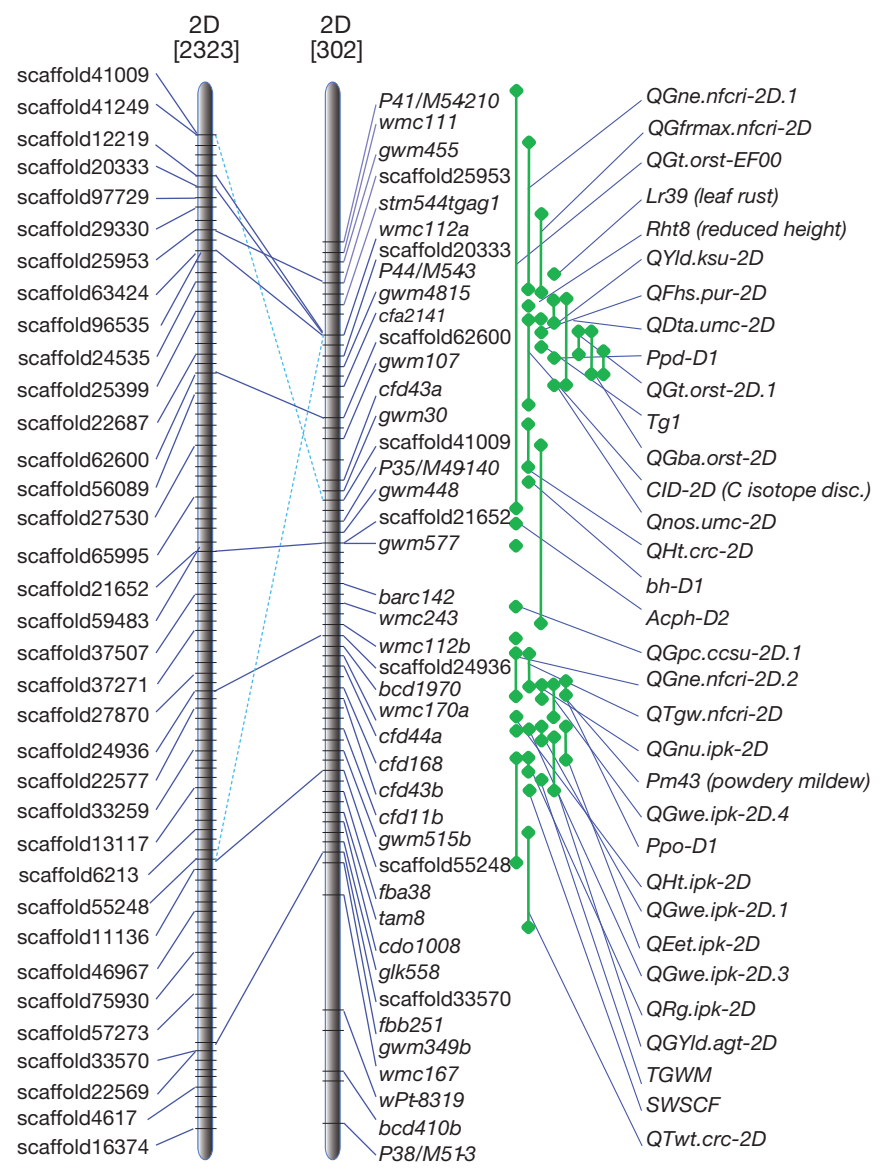

Figure $3 \mid$ An integrated genetic map of Ae. tauschii chromosome 2D. The Ae. tauschii genetic map was integrated with markers, scaffolds and mapped QTLs to assist in marker development and map-based cloning. Left: the Ae. tauschii molecular map used for synteny alignment in Fig. 1 was aligned to chromosome 2D (November 2011 consensus map, CMap; http:// ccg.murdoch.edu.au/cmap/ccg-live) where sequence information was available The original marker at a location is retained in CMap as a synonym. Right: within CMap, details for QTL locations are provided at a greater magnification to show all the markers in the regions of interest. The dotted lines indicate an ambiguous relationship that is most probably due to repetitive sequences.

\section{METHODS SUMMARY}

We selected Ae. tauschii $(2 n=14)$ accession AL8/78 for sequencing. Plants were grown at $25^{\circ} \mathrm{C}$ in a darkened chamber for two weeks; DNA was extracted from leaf tissue and purified with a standard phenol/chloroform extraction protocol. Sequencing libraries were constructed and sequenced on Illumina next-generation sequencing platforms (GAII and HiSequation (2000)). High-quality reads were assembled with SOAPdenovo ${ }^{3}$. Repeat sequences were identified by combining $d e$ novo approaches and sequence similarity at the nucleotide and protein levels. Gene models were predicted by combining homology-based, de novo and RNA-Seqbased methods. RNA-Seq reads were assembled with CAP3 (ref. 28) and CD-Hit ${ }^{29}$ and were mapped to the draft genome with Tophat ${ }^{30}$. See Supplementary Information for details and additional analyses.

Received 28 March 2012; accepted 20 February 2013.

Published online 24 March; corrected online 3 April 2013 (see full-text HTML version for details).

1. Salamini, F., Ozkan, H., Brandolini, A., Schafer-Pregl, R. \& Martin, W. Genetics and geography of wild cereal domestication in the near east. Nature Rev. Genet. 3 , 429-441 (2002).

2. Dubcovsky, J. \& Dvorak, J. Genome plasticity a key factor in the success of polyploid wheat under domestication. Science 316, 1862-1866 (2007)

3. Li, R. et al. De novo assembly of human genomes with massively parallel short read sequencing. Genome Res. 20, 265-272 (2010).

4. Salamov, A. A. \& Solovyev, V. V. Ab initio gene finding in Drosophila genomic DNA. Genome Res. 10, 516-522 (2000).
5. Wicker, T. et al. A whole-genome snapshot of 454 sequences exposes the composition of the barley genome and provides evidence for parallel evolution of genome size in wheat and barley. Plant J. 59, 712-722 (2009).

6. Williams, M. et al. Pliocene climate and seasonality in North Atlantic shelf seas. Phil Trans. R. Soc. A 367, 85-108 (2009).

7. Qi, L. L. et al. A chromosome bin map of 16,000 expressed sequence tag loci and distribution of genes among the three genomes of polyploid wheat. Genetics 168 , 701-712 (2004).

8. Poland, J. A., Brown, P. J., Sorrells, M. E. \& Jannink, J. L. Development of highdensity genetic maps for barley and wheat using a novel two-enzyme genotypingby-sequencing approach. PLOS ONE 7, e32253 (2012).

9. Mayer, K. F. et al. Unlocking the barley genome by chromosomal and comparative genomics. Plant Cell 23, 1249-1263 (2011)

10. The International Brachypodium Initiative. Genome sequencing and analysis of the model grass Brachypodium distachyon. Nature 463, 763-768 (2010).

11. McHale, L., Tan, X., Koehl, P. \& Michelmore, R. W. Plant NBS-LRR proteins: adaptable guards. Genome Biol. 7, 212 (2006).

12. Luo, S. et al. Dynamic nucleotide-binding-site and leucine-rich-repeat-encoding genes in the grass family. Plant Physiol. 159, 197-210 (2012).

13. Brenchley, R. et al. Analysis of the bread wheat genome using whole-genome shotgun sequencing. Nature 491, 705-710 (2012)

14. Yue, J. X., Meyers, B. C., Chen, J. Q., Tian, D. \& Yang, S. Tracing the origin and evolutionary history of plant nucleotide-binding site-leucine-rich repeat (NBSLRR) genes. New Phytol. 193, 1049-1063 (2012).

15. Schuler, M. A. \& Werck-Reichhart, D. Functional genomics of P450s. Annu. Rev. Plant Biol. 54, 629-667 (2003).

16. Thomashow, M. F. Molecular basis of plant cold acclimation: insights gained from studying the CBF cold response pathway. Plant Physiol. 154, 571-577 (2010)

17. Lata, C., Yadav, A. \& Prasad, M. in Abiotic Stress Response in Plants-Physiological, Biochemical and Genetic Perspectives (Shanker, A. \& Venkateswarlu, B., eds) 269-296 (InTech, 2011).

18. Masiero, S., Colombo, L., Grini, P. E., Schnittger, A. \& Kater, M. M. The emerging importance of type I MADS box transcription factors for plant reproduction. Plant Cell 23, 865-872 (2011).

19. Margolin, A. et al. ARACNE: an algorithm for the reconstruction of gene regulatory networks in a mammalian cellular context. BMC Bioinformatics 7 (Suppl. 1), S7 (2006).

20. Mao, X. et al. Transgenic expression of TaMYB2A confers enhanced tolerance to multiple abiotic stresses in Arabidopsis. Funct. Integr. Genomics 11, 445-465 (2011)

21. Hu, B. \& Chu, C. Phosphate starvation signaling in rice. Plant Signal. Behav. 6 , 927-929 (2011)

22. Shivaprasad, P.V.etal. A microRNA superfamily regulates nucleotide binding siteleucine-rich repeats and other mRNAs. Plant Cell 24, 859-874 (2012).

23. Gupta, R. B., Singh, N. K. \& Shepherd, K. W. The cumulative effect of allelic variation in LMW and HMW glutenin subunits on dough properties in the progeny of two bread wheats. Theor. Appl. Genet. 77, 57-64 (1989).

24. Chantret, N. et al. Molecular basis of evolutionary events that shaped the hardness locus in diploid and polyploid wheat species (Triticum and Aegilops). Plant Cell 17, 1033-1045 (2005).

25. Ravel, C. et al. Nucleotide polymorphism in the wheat transcriptional activator Spa influences its pattern of expression and has pleiotropic effects on grain protein composition, dough viscoelasticity, and grain hardness. Plant Physiol. 151, 2133-2144 (2009)

26. Talbert, L. E., Smith, L. Y. \& Blake, N. K. More than one origin of hexaploid wheat is indicated by sequence comparison of low-copy DNA. Genome 41, 402-407 (1998).

27. Trethowan, R. M. \& Mujeeb-Kazi, A. Novel germplasm resources for improving environmental stress tolerance of hexaploid wheat. Crop Sci. 48, 1255-1265 (2008).

28. Huang, X. \& Madan, A. CAP3: a DNA sequence assembly program. Genome Res. 9, 868-877 (1999).

29. Li, W. \& Godzik, A. Cd-hit: a fast program for clustering and comparing large sets of protein or nucleotide sequences. Bioinformatics 22, 1658-1659 (2006).

30. Trapnell, C., Pachter, L. \& Salzberg, S. L. TopHat: discovering splice junctions with RNA-Seq. Bioinformatics 25, 1105-1111 (2009).

Supplementary Information is available in the online version of the paper.

Acknowledgements We thank J. M. Wan for support and encouragement; J. Dvorak and M. C. Luo for the AL8/78 line; C. Y. Jin, X. Y. Li, L. C. Zhang, L. Pan and J. C. Zhang for material preparation; Y. H. Lv for providing helpful palaeogeological information; D. M. Appels for producing the CMap database of molecular genetic maps; $K$. Edwards for providing the details of the SNP-based map for Avalon X Cadenza; L. Goodman for assistance in editing the manuscript; and M. W. Bevan, Y. B. Xu and C. Zou for critical readings of the manuscript. This work was supported by grants from the National 863 Project (2012AA10A308 and 2011AA100104), the International S\&T Cooperation Program of China (2008DFB30080), the National Natural Science Foundation of China (31171548 and 31071415), the National Basic Research Program of China (2010CB125900), the Core Research Budget of the Non-profit Governmental Research (201013) and the National Program on R\&D of Transgenic Plants (2011ZX08009-001 and 2011ZX08002-002).

Author Contributions J.Z.J., Ji.W., Xu L., H.M.Y., Z.H.H., Lo.M., Ju.W., X.Y.K., X.Y.Z., R.L.J. and Y.Z.M. initiated the project and designed the study. X.Y.K., G.Y.Z., R.A., L.F.G., Ou.H. H.H.K., B.K., X.C.X., R.Z.Z. G.M.L. and C.M. performed the research. S.C.Z., Y.R.L., W.M.H., M.P., C.Z., D.L., C.G., M.S., K.M., Y.T., F.Y.Z., S.K.P., J.W.L., Q.S.L., Ji.C., C.Y.G., G.Y.H., Y.D.L, P.L., J.Y.W., J.Y.X. and J.L.G. generated and analysed the data. O.J.X., H.F.Z. and Z.W.Q. developed the high-density genetic map. J.Z.J., S.C.Z., Lo.M., R.A., G.Y.Z., X.Y.K. and T.W. wrote the paper. 
Author Information The genome sequence and the annotation are available from the National Centre for Biotechnology Information (NCBI) as BioProject ID PRJNA182898. This Whole Genome Shotgun project is deposited at DDBJ/EMBL/GenBank under accession number AOCO000000000. The version described in this paper is the first version, AOC0010000000. The Illumina sequencing reads are available in the Sequence Read Archive under accession number SRA030526, RNA-Seq sequences under SRA062662, and resequencing short reads under SRA063175. Genomic data are also available at the Comprehensive Library for Modern Biotechnology (CLiMB) repository under doi:10.5524/100054. Reprints and permissions information is available at www.nature.com/reprints. The authors declare no competing financia interests. Readers are welcome to comment on the online version of the paper. Correspondence and requests for materials should be addressed to Ju.W.

(wangj@genomics.cn), J.Z.J. (jzjia@mail.caas.net.cn), Lo.M. (maolong@caas.net.cn), Z.H.H. (zhhe@public3.bta.net.cn) and Xu L. (liuxu01@caas.cn).

(c) (i) This work is licensed under a Creative Commons Attribution-

Non Commercial-Share Alike 3.0 Unported licence. To view a copy of this licence, visit http://creativecommons.org/licenses/by-nc-sa/3.0 